Pacific Journal of Mathematic 


\section{RINGS WITH QUASI-PROJECTIVE LEFT IDEALS}

\section{S. K. Jain and SurJeet Singh}

A ring $R$ is a left $q p$-ring if each of its left ideals is quasi-projective as a left $R$-module in the sense of $W u$ and Jans. The following results giving the structure of left $q p$-rings are obtained. Throughout $R$ is a perfect ring with radical $N$ : (1) Let $R$ be local. Then $R$ is a left $q p$-ring iff $N^{2}=(0)$ or $R$ is a principal left ideal ring with $d c c$ on left ideals, (2) If $R$ is a left $q p$-ring and $T$ is the sum of all those indecomposable left ideals of $R$ which are not projective, then $T$ is an ideal of $R$ and $N=T \oplus L, L$ is a left ideal of $R$ such that every left subideal of $L$ is projective, $R / T$ is hereditary, and $R$ is heredity iff $T=(0)$. (3) If $R$ is left $q p$-ring then $R=\left(\begin{array}{cc}S & M \\ 0 & T\end{array}\right)$, where $S$ is hereditary, $T$ is a direct sum of finitely many local $q p$-rings and $M$ is a $(S, T)$-bimodule. (4) $\mathbf{A}$ perfect left $q p$-ring is semi-primary. (5) Let $R$ be an indecomposable ring such that it admits a faithful projective injective left module. Then $R$ is a left $q p$-ring iff $R$ is a local principal left ideal ring or $R$ is a left-hereditary ring with $d c c$ on left ideals. (6) Let $R$ be an indecomposable $Q F$-ring. Then $R$ is a left $q p$-ring if each homomorphic image of $R$ is a $q$-ring (each one-sided ideal is quasi-injective). (7) If a left ideal $A$ of left $q p$-ring $R$ is not projective then the projective dimension of $A$ is infinite, thus lgl. $\operatorname{dim} R=0,1$, or $\infty$. An example of a left artinian left $q p$-ring which is not right $q p$-ring is also given.

Clearly all left hereditary rings are left $q p$-rings. However, the class of commutative principal ideal artinian rings which are not direct sum of fields distinguishes $q p$-rings from hereditary rings. Commutative pre-self-injective rings studied by Klatt and Levy [8] and by Levy [11] form a class dual to the class of commutative $q p$-rings. Dual to the noncommutative $q p$-rings are rings for which every cyclic module is quasi-injective investigated by Ahsan [1] and by Koehler [9]. In this paper we study perfect left $q p$-rings.

2. A ring $R$ is said to be right (left) perfect if it satisfies $d c c$ on principal left (right) ideals and $R$ is called perfect if it is both right and left perfect [3]. An artinian principal ideal ring is called uniserial.

A ring $R$ with Jacobson radical $N$ is called local if $R / N$ is a division ring. We assume that all nonzero rings have nonzero identity elements 
and all modules are unital. An $R$-module $M$ is said to be quasiprojective if for every submodule $N$ of $M$, the induced sequence $0 \rightarrow \operatorname{Hom}(M, N) \rightarrow \operatorname{Hom}(M, M) \rightarrow \operatorname{Hom}(M, M / N) \rightarrow 0$ is exact. For basic properties of quasi-projective modules we refer to $\mathrm{Wu}$ and Jans [14]. Quasi-injective modules are defined dually in [7]. The following theorems give the structure of a quasi-projective module over a perfect ring.

Theorem 1. (Wu and Jans [14]). A finitely generated indecomposable quasi-projective left module over a right perfect ring $R$ is of the form $R e / A e$ where $e$ is a primitive idempotent and $A$ is an ideal of $R$ (Indeed the theorem is proved when $R$ is semi-perfect.).

Theorem 2. (Koehler [10]). Let $R$ be a right perfect ring. A left $R$-module $M$ is quasi-projective if and only if

$$
M=\bigoplus \sum_{i=1}^{k}\left(R e_{i} / A e_{i}\right)^{g(i)}
$$

Where $A$ is an ideal and $e_{1}, e_{2}, \cdots, e_{k}$ are indecomposable orthogonal idempotents; the number of nonisomorphic simple R-modules is $k$, and $R e_{1}, R e_{2}, \cdots, R e_{k}$ are the corresponding nonisomorphic projective covers. In addition the decomposition is unique upto automorphism.

As defined by Miyashita [12], a module $M$ is called perfect if for any pair of submodules $A, B$ of $M$ with $A+B=M$ there exists a submodule $B_{0}$ of $B$ that is minimal with respect to the property that $A+B_{0}=M$. In this case $B_{0}$ is called a $d$-complement of $A$ (in $M$ ).

THEOREM 3. (Miyashita [12]). If every homomorphic image of a module $M$ has a projective cover then $M$ is perfect. Further if $M$ is perfect and quasi-projective then the sum of two submodules of $M$ which are d-complements of each other is direct.

Finally, in this section we state a lemma which is analogous to the lemma in Rangaswamy and Vaneja [13].

Lemma 1. Let $A \oplus B$ be a quasi-projective left $R$-module. Then every epimorphism from $A$ to $B$ splits.

3. In all the Lemmas $2,3,4,5$ and 6 which follow it is assumed that $R$ is a perfect left $q p$-ring and we write $R=R e_{1} \oplus \cdots \oplus R e_{n}$, where $\left\{\boldsymbol{e}_{i}\right\}_{1 \leqq i \leqq n}$ are primitive orthogonal idempotents. Denote the Jacobson radical of $R$ by $N$. 
Lemma 2. Let $A$ and $B$ be two indecomposable left ideals of $R$. Then either $A \cap B=(0)$ or $A$ and $B$ are comparable.

Proof. Let $A \not \subset B$ and $B \not \subset A . \quad$ As $A+B$ is quasi-projective perfe ${ }^{*}$ ' 'eft $R$-module, by Theorem 3 , there exist nonzero left subideals $A_{0}$ of $A$ and $B_{0}$ of $B$ such that $A+B=A_{0} \oplus B_{0}$. Then $A=A_{0} \oplus\left(A \cap B_{0}\right)$ yields that $A=A_{0}$ as $A$ is indecomposable. Similarly $B=B_{0}$. Hence $A \cap B=(0)$.

LEMma 3. If an indecomposable left ideal $A$ is not projective then for some $i, A \subset N e_{i}$ and $A=R e_{i} x e_{i}$ for some $e_{i} x e_{i} \in e_{i} N e_{i}$; further for this $i, \quad R e_{i} R e_{j}=(0)$ for all $j \neq i$. In particular, if $e N e \neq(0)$ then hom $(R e, R f)=0$ for all primitive idempotents $f$ not equal to e. Also conversely, any left ideal of the form $A=R e_{i} x e_{i}, e_{i} x e_{i} \in e_{i} N e_{i}$ is an indecomposable nonprojective left ideal.

Proof. By Theorem 1, $A \cong R e_{i} / I e_{i}$ for some ideal $I$ of $R$. If $A \not \subset R e_{i}$, then by Lemma 2, $A \cap R e_{i}=(0)$. But then the left ideal $R e_{i} \oplus A$ of $R$ is quasi-projective and there exists an epimorphism $\sigma: R e_{i} \rightarrow A$ which must split by Lemma 1. So $\sigma$ is an isomorphism and $A$ is projective which is a contradiction. Hence $A \subset N e_{i}$, since $N e_{i}$ is the unique maximal left subideal of $R e_{i}$. Further, $A$ being a homomorphic image of $R e_{i}, A=R e_{i} x e_{i}$ for some $e_{i} x e_{i} \in e_{i} N e_{i}$. For proving $R e_{i} R e_{j}=0, i \neq j$, let us assume that for some $j, R e_{i} R e_{j} \neq 0$. So there exists $a \in R$ such that $R e_{i} a e_{j} \neq 0$. As $R e_{i} \oplus R e_{i} a e_{j}$ is quasiprojective, Lemma 1 yields that $R e_{i} \cong R e_{i} a e_{j}$. Then $R e_{i} a e_{j} \oplus A$ is quasi-projective and $A$ is a homomorphic image of $R e_{i} a e_{j}$. Consequently, Lemma 1 gives that $A$ is projective which is a contradiction. Hence for all $j \neq i, R e_{i} R e_{j}=(0)$.

LEMMA 4. For a fixed $i$, either the family of all nonzero left ideals of the form $R e_{i} a e_{i}, e_{i} a e_{i} \in e_{i} N e_{i}$ are isomorphic or $R e_{i} N e_{i}=R e_{i} n e_{i}$ for some $e_{i} n e_{i} \in e_{i} N e_{i}$.

Proof. Since $R$ is a perfect ring, $N$ is both right and left $T$-nilpotent. We assert that there exists a maximal left ideal in the family $F=\left\{R e_{i} x e_{i} \mid e_{i} x e_{i} \in e_{i} N e_{i}\right\} . \quad$ For if $R e_{i} b e_{i}$ is not maximal then we can find $R e_{i} b_{1} e_{i} \supset R e_{i} b e_{i}$. This gives $e_{i} b e_{i}=\left(e_{i} x e_{i}\right)\left(e_{i} b_{1} e_{i}\right)$ with $e_{i} x_{1} e_{i} \in e_{i} N e_{i}$. If $R e_{i} b_{1} e_{i}$ is not maximal then we can find $R e_{i} b_{2} e_{i} \supset$ $R e_{i} b_{1} e_{i} \supset R e_{i} b e_{i}$. This yields $e_{i} b e_{i}=\left(e_{i} x_{2} e_{i}\right)\left(e_{i} b_{1} e_{i}\right)$ and thus $e_{i} b e_{i}=$ $\left(e_{i} x_{2} e_{i}\right)\left(e_{i} x_{1} e_{i}\right)\left(e_{i} b_{i} e_{i}\right)$. By continuing this process, we get a sequence $\left(e_{i} x_{j} e_{i}\right), j=1,2, \cdots$, with $e_{i} x_{j} e_{i} \in e_{i} N e_{i}$. Since $N$ is $T$-nilpotent this sequence cannot be infinite. Hence we can find a maximal left ideal, 
say, $R e_{i} n e_{t}$ in the family $F$. We claim that either $R e_{i} N e_{i}=R e_{i} n e_{i}$ or all left ideals of the form $R e_{i} a e_{i}, e_{i} a e_{i} \in e_{i} N e_{i}$ are isomorphic. So if $R e_{i} N e_{i} \neq R e_{i} n e_{i}$ then there exists some $x \in . N$ such that $R e_{i} x e_{i} \not \subset R e_{i} n e_{i}$. Then by Lemma 2, $R e_{i} x e_{i} \cap R e_{i} n e_{i}=(0)$. Let $A=$ $R e_{i} n e_{i} \oplus R e_{i} x e_{i}$. A is a quasi-projective left ideal of $R$ and both $R e_{i} n e_{i}$, $R e_{i} x e_{i}$ have same projective cover $R e_{i}$. So by Theorem 2, $R e_{i} n e_{i} \cong$ $R e_{i} x e_{i}$. We now show for every $a \in N, R e_{i} a e_{i}$ is isomorphic to $R e_{i} n e_{i}$. By Lemma 2 and maximality of $R e_{i} n e_{i}, R e_{i} a e_{i}$ must have zero intersection with one of the two left ideals $R e_{i} n e_{i}, R e_{i} x e_{i}$. In either case we get by invoking Theorem 2 again that $R e_{i} a e_{i} \cong R e_{i} n e_{i}$. Hence all left ideals of the form $R e_{i} a e_{i}$ are isomorphic as desired. This completes the proof.

LEMMA 5. For a fixed $i$ either $\left(e_{i} N e_{i}\right)^{2}=(0)$ or $e_{i} R e_{i}$ is a principal left ideal ring with dcc (all proper left ideals are powers of $e_{i} N e_{i}$ ) and all left subideals of $R_{e_{i}}$ generated by subsets of $e_{i} N e_{i}$ satisfy dcc.

Proof. There is a 1-1 inclusion preserving correspondence between all left ideals of $e_{i} R e_{i}$ and all those left subideals of $R e_{i}$ which are generated by subsets of $e_{i} N e_{i}$. If, as in the Lemma 4, all nonzero principal left subideals of $R e_{i}$ of the form $R e_{i} a e_{i}, e_{i} a e_{i} \in e_{i} N e_{i}$ are isomorphic, we derive that all the principal left subideals of $e_{i} N e_{i}$ in $e_{i} R e_{i}$ are isomorphic and hence minimal. Consequently, $\left(e_{i} N e_{i}\right)^{2}=(0)$. In the other case we have $R e_{i} N e_{i}=R e_{i} n e_{i}$. This implies $e_{i} R e_{i} N e_{i}=e_{i} R e_{i} n \dot{e}_{i}$ and so $e_{i} N e_{i}=e_{i} R e_{i} n e_{i}$. Thus in the local ring $e_{i} R e_{i}$, the radical is a principal left ideal generated by a nilpotent element. This yields that all the left ideals of $e_{i} R e_{i}$ are of the form $e_{i} R e_{i}\left(e_{i} n e_{i}\right)^{t}\left(=\left(e_{i} N e_{i}\right)^{t}\right)$, $t=1,2, \cdots, k$, where $k$ is the index of nilpotency of $e_{i} N e_{i}$. But then this gives that all the left subideals of $R e_{i}$ generated by the subsets of $e_{i} N e_{i}$ are of the form $R\left(e_{i} n e_{i}\right)^{t}$. This completes the proof.

THEOREM 4. Let $R$ be a perfect left qp-ring. Then for any primitive idempotent $e$ of $R, e R e$ is also a left qp-ring.

Proof. Let $R=R e_{1} \oplus \cdots \oplus R e_{n}$, where $e_{i}$ are primitive orthogonal idempotents. Without loss of generality we can suppose that $e=$ $e_{1}$. Let $N=J(R)$ be the Jacobson radical. If $\left(e_{1} N e_{1}\right)^{2}=(0)$, then $e_{1} N e_{1}$ is a completely reducible left $e_{1} R e_{1}$-module. Trivially then every left ideal of $e_{1} R e_{1}$ is quasi-projective. Suppose $\left(e_{1} N e_{1}\right)^{2} \neq 0$. By Lemma 5, any proper left-ideal of $e_{1} R e_{1}$ is a power of $e_{1} N e_{1}$, and thus it is isomorphic to $e_{1} R e_{1} /\left(e_{1} N e_{1}\right)^{t}$ for some positive integer $t$ which is quasi-projective. Hence $e_{1} R e_{1}$ is a left $q p$-ring.

Combining Theorem 4 and the above lemmas we obtain: 
THEOREM 5. Let $R$ be a local perfect ring. Then $R$ is a left qp-ring if and only if

(i) $N^{2}=(0)$, or

(ii) $R$ is a principal left ideal ring with dcc on left ideals.

Next we prove a proposition which is also of an independent interest.

Proposition 1. Let $R$ be a left perfect ring. If every left ideal contained in the radical $N$ is projective, then $R$ is left hereditary.

Proof. Since idempotents modulo the radical can be lifted, given any left ideal $I$ of $R, I=R f_{1} \oplus \cdots \oplus R f_{n} \oplus J$, for some idempotents $f_{1}, \cdots, f_{n}$ and for some left ideal $J \subset N$. By hypothesis $J$ is projective. Hence $I$ is projective and so $R$ is left hereditary.

Lemma 6. Any nonzero left subideal of $\mathrm{Ne}$ ( $e$ primitive idempotent) of the form Reae in a perfect ring $R$ cannot have nonzero homorphism into any indecomposable left ideal $B$ which is a homomorphic image of some $R f$ with $f$, a primitive idempotent, such that $R f \neq R e$.

Proof. Let $A=$ Reae. Since $e N e \neq(0)$, by Lemma 3, ReRf $=(0)$, where $f$ is a primitive idempotent not equal to $e$. Since $A$ is not projective, each of its nonzero homomorphic image is also not projective. So let $B$ be an indecomposable homomorphic image of $A=$ Reae. Since $B$ is an indecomposable quasi-projective (but not projective) left ideal, by theorem $1, B$ is of the form $R f / X f$ where $X f \neq 0$ and $f$ is some primitive idempotent. We wish to show that $f=e$. By Lemma 3, $B \subset R f$. But then we get a nonzero homomorphism $R e \rightarrow R e a e \rightarrow B \rightarrow R f$ which is a contradiction unless $e=f$. Thus Reae cannot map onto any $R f / X f$ with $R f \neq R e$. This completes the proof.

THEOREM 6. Let $R$ be a perfect left qp-ring and let $e_{i}, 1 \leqq i \leqq n$, be a maximal set of primitive orthogonal idempotents in $R$. Suppose $T=$ $\sum_{i=1}^{n} R e_{i} N e_{i}$. Then (i) $T$ is the sum of all those indecomposable left ideals of $R$ which are not projective, (ii) $T$ is an ideal of $R$ contained in $N$, and (iii) $N=T \oplus L$ for some left ideal $L$ of $R$ such that every left subideal of $L$ is projective.

Proof. By Lemma 6, $R e_{i} N e_{i} R e_{j}=(0)$ for $i \neq j$. So $T$ is an ideal of $R$. Also, by Lemma 3, an indecomposable left ideal $A$ of $R$ is not projective if and only if $A=R e_{i} a e_{i}$ for some $0 \neq e_{i} a e_{i} \in e_{i} N e_{i}$. Thus it is immediate that $T$ is the sum of all nonprojective indecomposable left 
ideals of $R$. We now proceed to prove (iii). Since $N e_{i}$ is quasiprojective, we can write $N e_{i}=\bigoplus \Sigma B_{k}$, where $B_{k}$ are indecomposable left subideals of $N e_{i}$ (Theorem 2). Consider $0 \neq e_{i} x e_{i} \in e_{i} N e_{i}$. Then $R e_{i} x e_{i}$ has nonzero projection into some $B_{k}$. By Lemma $6, B_{k}$ itself is of the type $R e_{i} y e_{i}, e_{i} y e_{i} \in e_{i} N e_{i}$. It follows from Lemma 3 that $R e_{i} N e_{i}$ is a sum of those indecomposable left ideals $B_{k}$ which are homomorphic immages of $R e_{i}$. Also if some $B_{k}$ is not homomorphic image of $R e_{i}$, then this $B_{k}$ must be projective. Hence we can write $N e_{i}=R e_{i} N e_{i} \oplus C_{i}$ where $C_{i}$ is projective. This gives $N=T \oplus C$ where $C$ is projective. Consider a left ideal $B(\neq 0)$ contained in $C$. Now $B=$ $\oplus \Sigma X_{\alpha}$, where $X_{\alpha}$ are indecomposable left ideals. If some $X_{\alpha}$ is not projective, then by Lemma 3, $X_{\alpha}$ is of type $R e_{i} x e_{i}$ with $e_{i} x e_{i}$ in $e_{i} N e_{i}$ and hence $X_{\alpha} \subset T$ which is a contradiction. This shows that $B$ is projective, thus proving the theorem.

THEOREM 7. Let $R$ be a perfect left qp-ring, and $T$ be the ideal as in Theorem 6. Then $R / T$ is left hereditary and $R$ is left hereditary if $T=(0)$.

Proof. Consider a left ideal $A / T \subseteq N / T$. Since $N=T \oplus C$, we get $A=T \oplus(A \cap C)$. But all left subideals of $C$ are projective. So $A \cap C$ is projective as a left $R$-module. Also $T(A \cap C)=(0)$ gives that $A \cap C$ is projective as left $R / T$-module. Then by Proposition 1 , $R / T$ is left hereditary. The last assertion in the theorem is obvious. This completes the proof.

The next theorem gives us a representation of a perfect left $q p$-ring as a triangular matrix ring.

TheORem 8. Let $R$ be a left, right perfect left qp-ring. Then

(1) $R$ is semi-primary

(2) $R$ is an upper-triangular matrix ring of the form

$$
\left(\begin{array}{ll}
S & M \\
0 & T
\end{array}\right)
$$

where $S$ is a hereditary semi-primary ring, $T$ is a finite direct sum of local left qp-rings, and $M$ is an $(S, T)$-bimodule such that ${ }_{S} M$ is projective.

Before we prove this theorem we establish some preliminaries and prove three lemmas. Let $R$ be a perfect left $q p$-ring and $N$ be its radical. Let $R f_{1}, R f_{2}, \cdots, R f_{m}$ be a maximal set of nonisomorphic indecomposable left ideals of $R$ generated by primitive idempotents. By invoking Lemma 1 we note that any nonzero $R$ - 
homomorphism of $R f_{i}$ into $R f_{j}$ is a monomorphism. Define a relation $\leqq$ in the set $\left\{R f_{1}, \cdots, R f_{m}\right\}$ as follows: $R f_{i} \leqq R f_{j}$ if and only if there exists a nonzero $R$-homomorphism of $R f_{i}$ into $R f_{j}$, that is, $f_{i} R f_{j} \neq(0)$. By using the fact that in a right perfect ring $R$ no principal left ideal $R a$ of $R$ can be isomorphic to its own proper left subideal, we get that $\left\{R f_{1}, \cdots, R f_{m}\right\}$ is a partially ordered set with respect to $\leqq$. Further, recall Lemma 3 which says that for a given primitive idempotent $f$, either all left subideals of $R f$ are projective or $f N f \neq(0)$ and for any primitive idempotent $e$ with $R f \neq R e, f R e=(0)$. So if for some $f_{i}, R f_{i}$ has a left subideal which is not projective then $R f_{j} \not R f_{i}$ for all $i \neq j$. Hence we can arrange $R f_{1}, \cdots, R f_{m}$ in such a way that there exists a positive integer $u$ (possibly zero) which is less than or equal to $m$ satisfying the following:

(i) $\quad f_{j} R f_{i}=(0)$ for $i<j$.

(ii) Every left subideal of $R f_{i}$ is projective and $f_{i} R f_{i}$ is a division ring for $i \leqq u$.

(iii) $f_{j} N f_{j} \neq(0)$ and $f_{j} R f_{i}=(0)$ for $j>u$ and $i \neq j$.

Write

$R=\left(R f_{11} \oplus \cdots \oplus R f_{1 t_{1}}\right) \oplus\left(R_{21} \oplus \cdots \oplus R f_{2 t_{2}}\right) \oplus \cdots \oplus\left(R f_{m_{1}} \oplus \cdots \oplus R f_{m t_{m}}\right)$

where $f_{i j}$ are orthogonal primitive idempotents with their sum equal to 1 such that $R f_{i k} \cong R f_{i}$ for every $k$ and $i$. Clearly, by what is stated above, $t_{i}=1$ for $i \geqq u+1$; and $f_{i k} R f_{i k}$ is a division ring whenever $i \leqq u$. Let $E_{i}=\sum_{k=1}^{t_{i}} f_{i k}, 1 \leqq i \leqq m$ and $E=\sum_{i=1}^{u} E_{i}$. Then we have the following:

LEMmA 7. (1) For $i \leqq u, E_{i} R E_{i}$ is simple artinian.

(2) $E_{j} R E_{i}=(0)$ whenever $i<j$.

(3) $N$ is nilpotent.

Proof. Since $R f_{i k} \cong R f_{i}, 1 \leqq k \leqq t_{i}$ and $R E=\bigoplus \sum_{k=1}^{u} R f_{i k}$, we get $E_{i} R E_{t}$ is anti-isomorphic to the $t_{i} \times t_{i}$ matrix ring $D_{t_{t}}^{(i)}$ where $D^{(i)}=f_{i} R f_{i}$ is a division ring. This proves (1).

The proof of (2) is immediate consequence of the fact that $f_{j} R f_{i}=(0)$ for $i<j$.

Finally, to prove (3), let $A=\sum_{1<j} E_{i} R E_{j}$. Then $A$ is a nilpotent ideal and

$$
\begin{aligned}
R / A & \cong \oplus \sum_{i=1}^{u} E_{i} R E_{i} \oplus \sum_{i=u+1}^{m} E_{i} R E_{i} \\
& =\bigoplus \sum_{i=1}^{u} E_{i} R E_{i} \bigoplus_{i=u+1}^{m} f_{i 1} R f_{i 1} .
\end{aligned}
$$

Since each $E_{i} R E_{i}, 1 \leqq i \leqq u$, is simple artinian and by Theorems 4 and 5 each $f_{i 1} R f_{i 1}, u+1 \leqq i \leqq m$ is a local ring with nilpotent maximal 
ideal, we obtain that the radical of $R / A$ is nilpotent. Hence $N$ is nilpotent since $A$ is nilpotent.

\section{LEMMA 8. $S=E R E$ is hereditary.}

Proof. Since $V=\sum_{i<1 \leqq u} E_{i} R E_{j}$ is the radical of $S$ and $S$ is semiprimary, in order to prove $S$ is hereditary it is enough to prove that ${ }_{s} V$ is projective. Now

$$
V=\bigoplus \sum_{i<j \leqq u} E_{i} N E_{j}=\bigoplus \sum_{j=1}^{u} E N E_{j}=\bigoplus \sum_{k} \sum_{j=1}^{u} E N f_{j k} .
$$

Also by our arrangement $N f_{j k}$ is projective as left $R$-module whenever $j \leqq u$. Thus $E N f_{j k}$ is projective as left $E R E$-module and hence ${ }_{s} V$ is projective as desired.

LeMmA 9. $M=E R(1-E)$ is a projective left ERE-module.

Proof. Consider

$$
A=R E R(1-E)=\sum_{\alpha} \sum_{i \leq u} \sum_{k} R f_{i k} a,
$$

$a \in R(1-E)$. Hence $A$ is a homomorphic image of a projective module $P=\bigoplus \Sigma_{a \in E R(1-E)} \sum_{k} \Sigma_{i \leqq u} X_{i k a}$ where $X_{i k a} \cong R f_{i k}$ for $a \in R(1-E)$. Now $A$ has a projective cover $Q=\bigoplus \Sigma_{\alpha \in \wedge} X_{\alpha}$ such that each $X_{\alpha} \cong R f_{i(\alpha)}$, $1 \leqq i(\alpha) \leqq m$. As $A$ is a left ideal of $R, A$ is quasi-projective. So by Koehler's theorem (Theorem 2) there exists an ideal $B \subset N$ such that $A=\bigoplus \Sigma_{\alpha \in \wedge} Y_{\alpha}, Y_{\alpha} \cong(R / B) \bar{f}_{i(\alpha)}$. Since $Q$ is a projective cover of $A, Q$ is a direct summand of $P$. Thus, for each $i(\alpha)$, there exists a nonzero $R$-homomorphism of $R f_{i(\alpha)}$ into one of $R f_{i}$ with $i \leqq u$. This along with (2) of Lemma 7 yields that $i(\alpha) \leqq u$ for all $\alpha$. Since $Y_{\alpha} \subseteq R(1-E)$ and $i(\alpha) \leqq u$, an application of the Lemma 1 gives that the canonical homomorphism $R f_{i(\alpha)} \rightarrow(R / A) \bar{f}_{i(\alpha)}=Y_{\alpha}$ is an isomorphism. Hence $Y_{\alpha} \cong X_{\alpha}$ for all $\alpha$ and $A$ is projective.

Proof of the Theorem 8. Since $N$ is nilpotent, $R$ is semiprimary. Further, write

$$
R=E R E \oplus E R(1-E) \oplus(1-E) R(1-E) .
$$

By the above lemmas $S=E R E$ is hereditary and $M=E R(1-E)$ is a projective left $S$-module. Also $T=(1-E) R(1-E)=\bigoplus \sum_{i=u+1}^{m} f_{i} R f_{i}$ is a direct sum of local left $q p$-rings. Hence $R \cong\left(\begin{array}{ll}S & M \\ 0 & T\end{array}\right)$ where $S, T$ and $M$ are as stated in the theorem. 
4. In this section we prove a theorem for a perfect left $q p$-ring which admits a uniform projective left module. This theorem then enables us to characterize perfect left $q p$-rings which admit a faithful projective injective left module (Theorem 10). We begin with

THEOREM 9. Let $M$ be a uniform projective left module over a perfect left qp-ring $R$. Then $M \cong R e_{i}$ for some primitive idempotent $e_{i}$, and either (i) All left subideals of $\mathrm{Ne}_{1}$ are homomorphic image of $R_{e_{1}}$ and $\boldsymbol{R e}_{i} \boldsymbol{R e}_{j}=(0)=\operatorname{Re}_{j} \boldsymbol{R e}_{\mathrm{t}_{1}}$ where $e_{j}$ is a primitive idempotent such that $R e_{i} \neq R e_{j}$, or (ii) $N e_{1}$ is projective and all its left ideals are projective. In each case $R_{i}$ satisfies dcc on left subideals.

Proof. $\quad M \cong R e_{i}$ follows from well known result of Bass [3]. As in the proof of Theorem 6, we can write $N e_{i}=R e_{i} N e_{i} \oplus B_{1}$ where $B_{1}$ is projective. Since $R e_{1}$ is uniform either $N e_{i}=R e_{i} N e_{i}$ or $N e_{i}=B_{1}$. In case $N e_{i}=R e_{i} N e_{i}$, there exists $e_{i} n e_{i} \in e_{i} N e_{i}$ such that $N e_{i}=$ $R e_{i} n e_{i}$. Since $e_{i} n e_{i}$ is nilpotent, we get that every left subideal of $R e_{i}$ is of the form $R\left(e_{i} n e_{t}\right)^{t}=\left(R e_{i} n e_{i}\right)^{t}$ which is obviously a homomorphic image of $R e_{i}$. It also follows that $R e_{t}$ has only a finite number of left subideals. By Lemma 3, we know $R e_{i} R e_{j}=(0)$ where $R e_{i} \neq R e_{j}$. We show that $R e_{j} R e_{i}$ is also zero. Suppose not then we can choose $x e_{i} \in R e_{1}$ with $R e_{j} x e_{i} \neq 0$ and $R e_{j} x e_{i} \subset R e_{i}$. Lemma 1 yields that $R e_{j} x e_{i}$ is projective and thus $R e_{j} x e_{i} \cong R e_{i}$. But this is a contradiction since $R$ is perfect. This proves (i).

( In the other case we have $N e_{i}=B_{1}$ and $B_{1}$ is projective (also uniform). So $B_{1}$ is isomorphic to some $R e_{j}$. Also by theorem 6 every subideal of $B_{1}$ is projective and hence isomorphic to some $R e_{k}$. Further by Lemma 2 it follows that the subideals of $N e_{i}$ are totally ordered. Since no subideal ( $\neq R e_{j}$ ) of $R e_{j}$ can be isomorphic to $R e_{j}$, we conclude that there are only a finite number of subideals of $N e_{i}$. This completes the proof.

The next theorem characterizes perfect left $q p$-rings admitting a faithful projective injective module.

THEOREM 10. Let $R$ be an indecomposable (as a ring) perfect ring such that it admits a faithful projective injective left $R$-module $M$. Then $R$ is a left qp-ring if and only if

(i) $R$ is a local principal left ideal ring, or

(ii) $R$ is a left hereditary ring with dcc on left ideals

Proof. Sufficiency is obvious. So let $R$ be a left $q p$-ring. If we write $R=R e_{1} \oplus \cdots \oplus R e_{n}, e_{i}$ primitive orthogonal idempotents, then by Bass [3] $M$ is a direct sum of copies of $R e_{i}$ 's, say, $R e_{t+1}, \cdots, R e_{n}$. Then 
$A=R e_{t+1} \oplus \cdots \oplus R e_{n}$ is a faithful injective projective left $R$ module. We claim that each $R e_{i}, 1 \leqq i \leqq n$, is uniform. If $i \geqq t+1$ then it is clear that $R e_{i}$ is uniform. So let $i<t$. As $A$ is faithful, $e_{i} R e_{j} \neq(0)$ for some $j \geqq t+1$. By using Lemma 1 , we get that $R e_{i}$ is isomorphic to a left subideal of $R e_{j}$. Hence $R e_{i}$ is uniform, since $R e_{j}$ is uniform.

Now by Theorem $9, R e_{i}$ satisfies $d c c$. It is also clear from the proof of that theorem that each of the left subideals in $R e_{i}$ is principal. Hence $R$ satisfies $d c c$ on left ideals. In case $n=1, R$ is of type (i). So consider the case when $n>1$. We claim $N e_{i}$ is projective. For if $N e_{i}$ is not projective, then by Theorem $9, R e_{i}$ and $\sum_{j \neq i} R e_{j}$ are two nonzero ideals and $R=R e_{i} \oplus \Sigma_{j \neq i} R e_{j}$. This contradicts the assumption that $R$ is indecomposable. Hence $N e_{i}$ is projective. So $N=\bigoplus \Sigma N e_{i}$ is projective as a left $R$-module. Hence $R$ is left hereditary left artinian. This completes the proof.

As a special case of the above theorem we have the following characterization of $Q F$-rings.

Theorem 11. Let $R$ be an indecomposable QF-ring. Then $R$ is a left qp-ring iff each homomorphic image of $R$ is a q-ring (each one-sided ideal is quasi-injective).

Proof. Since a left hereditary $Q F$-ring is semisimple artinian, Theorem 10 gives that either $R$ is simple or local uniserial. In a local uniserial ring every one sided ideal is two sided and every homomorphic image is $Q F$-ring. Consequently, every homomorphic image is a q-ring [6].

Conversely, if every homomorphic image of $R$ is a $Q$-ring then also $R$ is uniserial ( $R$ is uniserial iff every homomorphic image of $R$ is $Q F$, Fuller [5]). Further $R$ is isomorphic to a full $n \times n$ matrix ring over a local ring $B$. If $n=1$ then $R$ is local uniserial. If $n>1$ then $R$ must be simple artinian, since $R$ is a $q$-ring (c.f. Jain, Mohamed and Singh [6], Theorem 2.4) [6]. In each case $R$ is a left $q p$-ring. This completes the proof.

5. In this section we study left global dimension of a perfect left $q p$-ring.

THEOREM 12. Let $R$ be a perfect left qp-ring and $A$ be a left ideal of $R$. Then the projective dimension of $A$ as a left $R$-module is 0 or $\infty$.

Proof. We first prove a sublemma. 
SublemMA. Under the hypothesis of the theorem if $e$ is a primitive idempotent and $0 \neq$ exe $\in e N e$ and $1_{R}$ (exe) denotes the left annihilator of exe in $R$ then $1_{R}(\mathrm{exe})=L \oplus M$, where $L=$ Reye, $0 \neq$ eye $\in e N e$, is not projective.

Proof of the sublemma. By Theorem 2 we can write $1_{R}(\mathrm{exe})=$ $\oplus \Sigma A_{\alpha}$ where $A_{\alpha}$ are indecomposable left ideals. Also it follows from Lemma 5 that $1_{R}(\mathrm{exe}) \cap e N e \neq 0$. Let us choose $0 \neq e u e \in 1_{R}(\mathrm{exe}) \cap$ $e N e$. Then Reue has nonzero homomorphism into one of $A_{\alpha}$ 's. By Lemma $6, A_{\alpha}=$ Reye for some eye in $e R e$. Indeed eye $\in e N e$ since $\operatorname{Re} \not \subset 1_{R}$ (exe). Hence $A_{\alpha}$ is not projective. This completes the proof of the sublemma.

We now prove the theorem. Since $A$ is a direct sum of indecomposable left ideals (Theorem 2) we may assume that $A$ is a nonzero indecomposable left ideal. If $A$ is projective then the projective dimension is zero. So let $A$ be not projective. Then by Lemma 3, $A=$ Rexe for some $0 \neq e x e \in e N e$ ( $e$ being some primitive idempotent). We construct an infinite projective resolution of $A$

$$
\cdots P_{n} \stackrel{f_{n}}{\rightarrow} P_{n-1} \cdots \rightarrow P_{1} \stackrel{f_{1}}{\rightarrow} P_{0} \stackrel{f_{0}}{\rightarrow} A \rightarrow 0
$$

such that for every $n, \operatorname{ker} f_{n} \cong A_{n} \oplus B_{n}$ where $A_{n}$ is nonprojective indecomposable left ideal of $R$ and is of the form $\operatorname{Rex}_{n} e, 0 \neq e x_{n} e \in$ $e N e$. Choose $P_{0}=R e$ and let $f_{0}$ be the natural $R$-homomorphism of $R e$ onto Rexe. Then $\operatorname{ker} f_{0}=1_{R}(\mathrm{exe})=A_{0} \oplus B_{0}$ where $A_{0}=\operatorname{Rex} e_{0} e$ is not projective (sublemma). Suppose we have constructed $P_{0}, P_{1}, \cdots, P_{n}$ with exact sequence

$$
0 \rightarrow \operatorname{ker} f_{n} \stackrel{\lambda}{\rightarrow} P_{n} \stackrel{f_{n}}{\rightarrow} P_{n-1} \rightarrow \cdots P_{1} \rightarrow P_{0} \stackrel{f_{0}}{\rightarrow} A
$$

where $\lambda$ is injection. By induction hypothesis $\operatorname{ker} f_{n}=A_{n} \oplus B_{n}$, where $0 \neq A_{n}=\operatorname{Rex}_{n} e \subset N e$.

Consider short exact sequences

$$
0 \rightarrow 1_{R}\left(e x_{n} e\right) \stackrel{\sigma_{n}}{\rightarrow} R e \stackrel{\eta_{n}}{\rightarrow} A_{n} \rightarrow 0
$$

and

$$
0 \rightarrow D_{n+1} \stackrel{\sigma_{n}^{\prime}}{\rightarrow} Q_{n} \stackrel{\eta_{n}^{\prime}}{\rightarrow} B_{n} \rightarrow 0
$$

where $\eta_{n}$ is a natural $R$-homomorphism, $\sigma_{n}$ is an injection and $Q_{n}$ is some projective module. 
Set $\quad P_{n+1}=Q_{n} \oplus R e$ and $f_{n+1}=\lambda\left(\eta_{n}^{\prime} \oplus \eta_{n}\right)$. Then ker $f_{n+1}=$ $\operatorname{ker} \eta_{n}^{\prime} \oplus \operatorname{ker} \eta_{n}$. Also ker $\eta_{n}=1_{R}\left(e x_{n} e\right)=\operatorname{Rex}_{n+1} e \oplus K$ (by sublemma). Thus $f_{n+1}$ has the required property. Since $P_{n+1}$ is projective, we have obtained the desired projective resolution of $A$.

Recall that if $R$ is not a semisimple artinian ring then

l. $g l \operatorname{dim} R=1+\sup \left\{1 . \operatorname{dim}_{R} A \mid A\right.$ is a left ideal $\}$.

The previous theorem then yields the following

Theorem 13. Let $R$ be a perfect left qp-ring. Then l. $g l \operatorname{dim} R=0,1$, or $\infty$.

6. It is well known that a left hereditary semiprimary ring is also right hereditary [2]. Here we give an example of a local primary .ring which is a left $q p$-ring but is not a right $q p$-ring.

EXAMPLE. Let $F$ be a field which has an isomorphism $a \rightarrow \bar{a}$ that is not an automorphism, and let $\bar{F}$ be the subfield of the images $\bar{a}, a \in F$. Take $x$ to be an indeterminate over $F$. Let $F[x]$ be the ring of polynomials of the form $a_{0}+a_{1} x+a_{2} x^{2}, a_{i} \in F$; multiplication being defined by the rule $x a=\bar{a} x, x^{3}=0$ together with distributive law. It is well known that such rings are principal left ideal rings. Its radical $N=\left\{a_{1} x+a_{2} x^{2} \mid a_{i} \in F\right\}$ is such that $N^{2} \neq(0), N^{3}=(0)$ and is a maximal left ideal of $R$. So $R$ is a local perfect ring. Also $N$ is not principal as a right ideal. So by Theorem $5, R$ is a left $q p$-ring but not a right $q p$ - ring.

\section{REFERENCES}

1. J. Ahsan, Rings all of whose cyclic modules are quasi-injective, Proc. London Math. Soc., 27 (1973), 425-443.

2. M. Auslander, On the dimension of modules and algebras III, global dimension, Nagoya Math. J., 9 (1955), 67-77.

3. H. Bass, Finitistic dimension and a homological generalization of semiprimary rings, Trans. Amer. Math. Soc., 95 (1960), 466-488.

4. C. Faith, Algebra-Rings, Modules and Categories I., Springer Verlag (1973).

5. K. Fuller, Generalized uniserial rings and their Kupisch Series, Math. Zeitschr., 106 (1968), 248-260.

6. S. K. Jain, S. Mohamed, and S. Singh, Rings in which every right ideal is quasi-injective, Pacific J. Math., 31 (1969), 73-79.

7. R. E. Johnson and E. T. Wong, Quasi-injective modules and irreducible rings, J. London Math. Soc., 36 (1961), 260-268.

8. G. Klatt and L. Levy, Pre-self-injective rings, Trans. Amer. Math. Soc., 137 (1969), 407-419.

9. A. Koehler, Rings with quasi-injective cyclic modules, Quart. J. Math. Oxford, Ser. 2 (1974), 51-55.

10. Quasi-projective and quasi-injective modules, Pacific J. Math., 36 (1971), 713-720. 
11. L. Levy, Commutative rings whose homomorphic images are self-injective, Pacific J. Math., 18 (1966), 149-153.

12. Y. Miyashita, Quasi-projective modules, perfect modules, and a theorem for modular lattices, J. Fac. Sci. Hokkaido University, Ser. 119 (1966), 86-110.

13. K. Rangaswamy and N. Vaneja, Quasi-projectives in abelian and module categories, Pacific J. Math., 43 (1972), 221-238.

14. L. Wu and J. Jans, On quasi-projectives, Illinois J. Math., 11 (1967), 439-448.

Received February 11, 1974. A part of this work was done while Surjeet Singh was at Bedford College, London, under the Younger Scientists Exchange Programme between India and U.K.

OHio University, ATHENS, OHIO 45701

AND

Aligarh Muslim University, India. 

D. E. Bennett, Strongly unicoherent continua ............................. 1

Walter R. Bloom, Sets of p-spectral synthesis ................................ 7

R. T. Bumby and D. E. Dobbs, Amitsur cohomology of quadratic extensions: Formulas and number-theoretic examples ................. 21

W. W. Comfort, Compactness-like properties for generalized weak topological sums

D. R. Dunninger and J. Locker, Monotone operators and nonlinear biharmonic boundary value problems ...

T. S. Erickson, W. S. Martindale, 3rd and J. M. Osborn, Prime nonassociative algebras

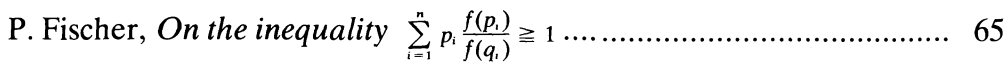

G. Fox and P. Morales, Compact subsets of a Tychonoff set ............... 75

R. Gilmer and J. F. Hoffmann, A characterization of Prüfer domains in terms of polynomials ......................................................... 81

L. C. Glaser, On tame Cantor sets in spheres having the same projection in each direction ......................................................... 87

Z. Goseki, On semigroups in which $X=X Y X=X Z X$ if and only if

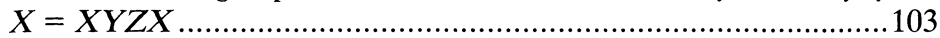

E. Grosswald, Rational valued series of exponentials and divisor

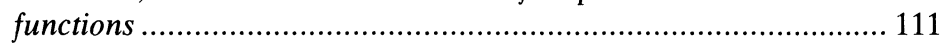

D. Handelman, Strongly semiprime rings ...................................... 115

J. N. Henry and D. C. Taylor, The $\bar{\beta}$ topology for $w^{*}$-algebras ............. 123

M. J. Hodel, Enumeration of weighted p-line arrays .......................... 141

S. K. Jain and S. Singh, Rings with quasiprojective left ideals .............. 169

S. Jeyaratnam, The diophantine equation $Y(Y+m)(Y+2 m) \times$

$$
(Y+3 m)=2 X(X+m)(X+2 m)(X+3 m) \ldots \ldots \ldots \ldots \ldots \ldots \ldots . . .183
$$

$\mathrm{R}$. Kane, On loop spaces without $p$ torsion .........................................189

Alvin J. Kay, Nonlinear integral equations and product integrals ..........203

A. S. Kechris, Countable ordinals and the analytic hierarchy, I ...........223

Ka-Sing Lau, A representation theorem for isometries of $C(X, E) \ldots \ldots . .229$

I. Madsen, On the action of the Dyer-Lashof algebra in $H_{*}(G)$..........235

R. C. Metzler, Positive linear functions, integration, and Choquet's theorem ........................................................................................ 277

A. Nobile, Some properties of the Nash blowing-up ............................297

G. E. Petersen and G. V. Welland, Plessner's theorem for Riesz conjugates 


\section{Pacific Journal of Mathematics}

\section{Vol. 60, No. $1 \quad$ September, 1975}

Donald Earl Bennett, Strongly unicoherent continua ................ 1

Walter Russell Bloom, Sets of p-spectral synthesis ................ 7

Richard Thomas Bumby and David Earl Dobbs, Amitsur cohomology of

quadratic extensions: formulas and number-theoretic examples .......

W. Wistar (William) Comfort, Compactness-like properties for generalized

weak topological sums ...................................

Dennis Robert Dunninger and John Stewart Locker, Monotone operators

and nonlinear biharmonic boundary value problems ..............

Theodore Erickson, Wallace Smith Martindale, III and J. Marshall Osborn,

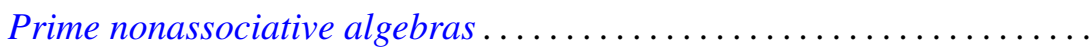

Pál Fischer, On the inequality $\sum_{i=0}^{n}\left[f\left(p_{i}\right) / f\left(q_{i}\right)\right] p_{i} \geq i \ldots \ldots \ldots \ldots \ldots$

Geoffrey Fox and Pedro Morales, Compact subsets of a Tychonoff set.......

Robert William Gilmer, Jr. and Joseph F. Hoffmann, A characterization of

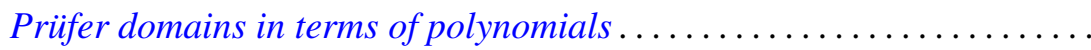

Leslie C. Glaser, On tame Cantor sets in spheres having the same projection

in each direction . ...................................

Zensiro Goseki, On semigroups in which $x=x y x=x z x$ if and only if

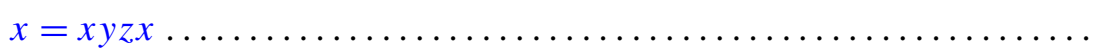

Emil Grosswald, Rational valued series of exponentials and divisor

functions.

David E. Handelman, Strongly semiprime rings

Jackson Neal Henry and Donald Curtis Taylor, The $\bar{\beta}$ topology for

$W^{*}$-algebras

Margaret Jones Hodel, Enumeration of weighted p-line arrays ...

Surender Kumar Jain and Surjeet Singh, Rings with quasi-projective left

ideals.

S. Jeyaratnam, The Diophantine equation

$$
Y(Y+m)(Y+2 m)(Y+3 m)=2 X(X+m)(X+2 m)(X+3 m) \ldots
$$

Richard Michael Kane, On loop spaces without $p$ torsion

Alvin John Kay, Nonlinear integral equations and product integrals ...

Alexander S. Kechris, Countable ordinals and the analytical hierarchy.

$I$.

Ka-Sing Lau, A representation theorem for isometries of $C(X, E)$

Ib Henning Madsen, On the action of the Dyer-Lashof algebra in $H_{*}(G)$

Richard C. Metzler, Positive linear functions, integration, and Choquet's

theorem.

Augusto Nobile, Some properties of the Nash blowing-up

Gerald E. Peterson and Grant Welland, Plessner's theorem for Riesz. 